\title{
ENQUETTE SUR LES SCHISTOSOMES DE L'HOMME ET DU BÉTAIL AU SENÉgAL, A L'AIDE DES IDENTIFICATIONS SPÉCifiques FOURnies PAR LA ChÉtotaxie DES CERCAIRES
}

\author{
I. Nouveaux arguments pour la validation \\ de S. curassoni Brumpt, 1931, parasite de l'Homme \\ et des Bovidés domestiques
}

\author{
J.-L. ALBARET*, H. PICOT**, 0. T. DIAW***, Ch. BAYSSADE-DUFOUR*, \\ G. VASSILIADES***, M. ADAMSON*, G. LUFFAU**** et A. G. CHABAUD* \\ Collaboration technique : Y. Sarr
}

RÉSUMÉ. Étude de nombreuses cercaires du Sénégal, provenant soit de Mollusques spontanément infestés, soit de Mollusques infestés avec des œufs prélevés dans des urines humaines ou dans des foies de Mouton et étude des cufs des Schistosomes correspondants.

La position relative des sensilles dorsales des cercaires fournit un "indice AD " et le rapport longueur sur largeur des œufs un " indice $L / l$ ".

Dans le stade actuel du travail, les données épidémiologiques sont incomplètes, certaines des données expérimentales sont imparfaites et l'analyse iso-enzymologique des adultes correspondant aux souches étudiées n'est pas encore faite.

Cependant, les données fournies par les indices $\mathrm{AD}$ et $\mathrm{L} / \mathrm{l}$ sont concordantes et conduisent à admettre l'existence d'une espèce différente de mattheei, de haematobium et de bovis, espèce qu'à la suite de Brumpt et de Grétillat, nous nommons Schistosoma curassoni.

Si l'on se fie à ces indices, l'enquête indique que :

a) S. mattheei n'a pas été trouvé au Sénégal, b) $S$. bovis y devient assez rare, c) S. haematobium semble exister à l'état pur en Casamance et serait mélangé à $S$. curassoni chez l'Homme dans le Nord et dans l'Est, d) $S$. curassoni semble l'espèce dominante chez les ruminants domestiques et chez l'Homme dans le Nord et dans l'Est, e) Bulinus umbilicatus serait le vecteur principal des 2 espèces.

\footnotetext{
* Laboratoire de Zoologie (Vers), associé au CNRS, Muséum National d'Histoire Naturelle, 61 rue de Buffon, F 75231 Paris Cedex 05.

** Parasitologie, Faculté de Médecine, 15 rue de l'Ecole-de-Médecine, F 75006, Paris.

*** Parasitologie, Laboratoire national de Recherches vétérinaires, B.P. 2057, Dakar, Sénégal.

**** Station de Recherche de Virologie et Immunologie, INRA, F 78850, Thiverval-Grignon.

Accepté le I 7 septembre I 984 .
} 
Investigations on Schistosomes of man and livestock in Senegal with the aid of cercarial chaetotaxy - I. New arguments for the validity of S. curassoni Brumpt, 1931, a parasite of man and domestic Bovidae

SUMMARY. Study of Senegal cercariae emitted by naturally infected snails or by snails experimentally infected using eggs from human urine or from sheep liver, and study of the corresponding eggs.

The relative position of the dorsal sensillae of cercariae is described by an "AD index" and the ratio length/width of eggs by an "L/l index".

At the present time, we have not finished the epidemiological studies, certain experiments are imperfect and the isoenzyme analysis of the corresponding adults is incomplete. Nevertheless information given by the indices $\mathrm{AD}$ and $\mathrm{L} / \mathrm{l}$ agree and lead us to admit the existence of a Schistosome different to mattheei, haematobium and bovis and which, following Brumpt and Gretillat we refer to as Schistosoma curassoni.

If one relies on the indices, the study indicates that:

a) S. mattheei has not been found in Senegal, b) S. bovis is quite rare, c) S. haematobium apparently exists in pure infections in Casamance and in mixed infections with S. curassoni in man in the north and in the east, d) S. curassoni is apparently the dominant species in domestic ruminants and in man in the north and in the east, e) Bulinus umbilicatus is the principal vector of $S$. curassoni and S. haematobium.

\section{Introduction}

Un point important dans l'épidémiologie de la Schistosomose est de pouvoir différencier les cercaires des différentes espèces et, en particulier, celles de $S$. bovis et celles de $S$. haematobium.

Les données fournies par les travaux de Richard (1971) font supposer que ce problème peut être résolu par la chétotaxie des cercaires. L'auteur montre que, sur les 2 souches dont elle dispose, les formes larvaires de Schistosoma haematobium et $S$. bovis peuvent être distinguées par la position relative des papilles AID, AIID et AIIID.

Cette donnée est reprise et élargie par Bayssade-Dufour (1982), qui étudie les chétotaxies cercariennes de 10 espèces de Schistosomes.

Nous avons essayé de vérifier ce résultat par l'étude du caractère (nommé ici indice $\mathrm{AD}$ ) sur différentes souches déjà déterminées et sur des souches provenant de deux missions effectuées au Sénégal.

La différence entre l'indice $\mathrm{AD}$ de $S$. bovis et de $S$. haematobium se confirme dans toutes les nouvelles données disponibles, mais la question est plus complexe, car il apparait que les indices $\mathrm{AD}$ de ce que nous considérions comme $S$. haematobium paraissent se répartir eux-mêmes en 3 groupes distincts.

Les mensurations faites sur les œufs correspondant à ces trois types de souches montrent, elles aussi, que les oufs se répartissent en différents groupes, dont les rapports longueur/largeur (L/l) sont significativement distincts. 
Les indices AD des cercaires et $\mathrm{L} / \mathrm{l}$ des œufs montrent qu'il ne s'agit pas de S. mattheei.

Dans le stade actuel du travail, les données épidémiologiques sont incomplètes, certaines des données expérimentales sont imparfaites et l'analyse iso-enzymologique des adultes correspondant aux souches étudiées n'est pas encore faite.

Cependant, les données fournies par les indices $\mathrm{AD}$ et $\mathrm{L} / \mathrm{l}$ sont si concordantes et conduisent si clairement à l'hypothèse de la revalidation de $S$. curassoni que nous pensons qu'il est utile de publier immédiatement les résultats obtenus.

\section{I - Méthodes}

\section{1 - Indice AD des cercaires}

Les données fournies par J. Richard (1971), complétées par celles de BayssadeDufour (1982), montrent que l'indice $\mathrm{AD}$ des cercaires constitue un caractère très intéressant pour l'identification des Schistosomes.

L'indice $\mathrm{AD}$ est le rapport qui correspond à la distance entre les papilles $\mathrm{AID}$ AIID sur la distance entre les papilles AIID-AIIID.

La mise en évidence des soies est réalisée en plongeant les cercaires vivantes dans une solution de nitrate d'argent à $2 \%$. Après réduction de l'argent par l'exposition aux rayons ultra-violets (ou au soleil en pays chaud) et lavage à l'eau distillée, les cercaires sont montées dans la gomme au chloral (technique simplifiée de Combes et coll., 1976).

\section{2 - Rapport $L / 1$ des aufs}

Pitchford (1965) a montré tout l'intérêt de la morphologie des aufs pour la détermination des Schistosoma à éperon terminal.

Seuls les œufs extra-utérins contenant un miracidium bien formé sont utilisés. Le rapport Longueur (L) sur largeur (l) fournit les données les plus nettes pour séparer les différents matériaux.

L'intervalle de confiance est déterminée à $5 \%$ sur la valeur de la moyenne (cas des petits échantillons, Schwartz, 1963, p. 154).

Dans quelques cas où les intervalles de confiance se chevauchent, un test de $t$ (Schwartz, 1963, p. 158) est utilisé pour savoir si les moyennes sont significativement différentes (risque à $5 \%$ ).

Lorsque le nombre d'œufs mesurés est très insuffisant, la donnée est fournie à simple titre indicatif.

\section{3 - Caractères divers}

\section{- Caractères biochimiques}

Les spécimens adultes ou immatures obtenus au cours du travail sont conservés dans l'azote liquide. L'analyse iso-enzymologique sera faite ultérieurement. 


\section{- Hôtes définitifs}

Les autopsies d'animaux domestiques spontanément infestés ont été faites aux abattoirs de Dakar ou de Tambacounda. A Dakar, les animaux proviennent de la moitié Nord du pays.

L'expérimentation porte sur différents Mammifères : Souris, Hamster, Rongeurs divers en élevage à Paris.

En ce qui concerne les Moutons, l'expérimentation faite à Dakar n'a qu'une valeur indicative puisque les animaux sont achetés au marché local et peuvent avoir une infestation naturelle. L'expérimentation faite dans la région parisienne, au contraire, est valable.

Les urines humaines contenant des œufs sont celles d'écoliers de tel ou tel village. Il faut noter qu'il s'agit d'un mélange des urines de plusieurs enfants.

\section{- Mollusques}

Dans la plupart des cas, l'expérimentation sur Mollusques n'est pas rigoureuse, car les spécimens soumis à l'infestation expérimentale sont récoltés dans la nature et peuvent avoir une infestation spontanée.

Les Mollusques ainsi récoltés sont surveillés quelques jours et ceux qui émettent spontanément des cercaires sont progressivement éliminés, mais le délai entre la date de récolte et la date d'utilisation pour l'infestation a souvent été trop courte (environ 10 jours) pour éliminer toute cause d'erreur bien que la surveillance ait été poursuivie environ 20 jours après l'infestation expérimentale pour procéder éventuellement à l'élimination des Mollusques émettant prématurément des cercaires.

Les déterminations des Mollusques ont été vérifiées soit à Copenhague par le Dr. F. Frandsen, soit à Londres par le Dr. D. S. Brown.

\section{- Localisations géographiques}

Lés Mollusques ont été récoltês au cours de deux missions : l'une dans le Nord (région de Matam) et dans l'Est (région de Tambacounda) en nov.-déc. 1983, l'autre dans le Sud (région de Kolda) en janvier-février 1984.

Le nom des localités est indiqué sur la figure 1.

\section{- Caractères des gîtes}

Les gîtes ont été choisis pour avoir soit une fréquentation humaine (petites mares des villages), soit une fréquentation animale (grandes étendues d'eau loin des villages).

Cette distinction est en fait assez illusoire. Les grandes étendues d'eau sont asséchées ; les grands Bovins viennent boire dans les villages et les enfants fréquentent tous les points d'eau, situés loin ou près des villages.

Il semble que Schistosoma bovis soit de plus en plus rare, car les gros Bovins qui boivent dans de petits abreuvoirs ne contaminent pas l'eau avec leurs déjections, comme ils le faisaient dans les grandes mares lorsque l'eau était abondante. 


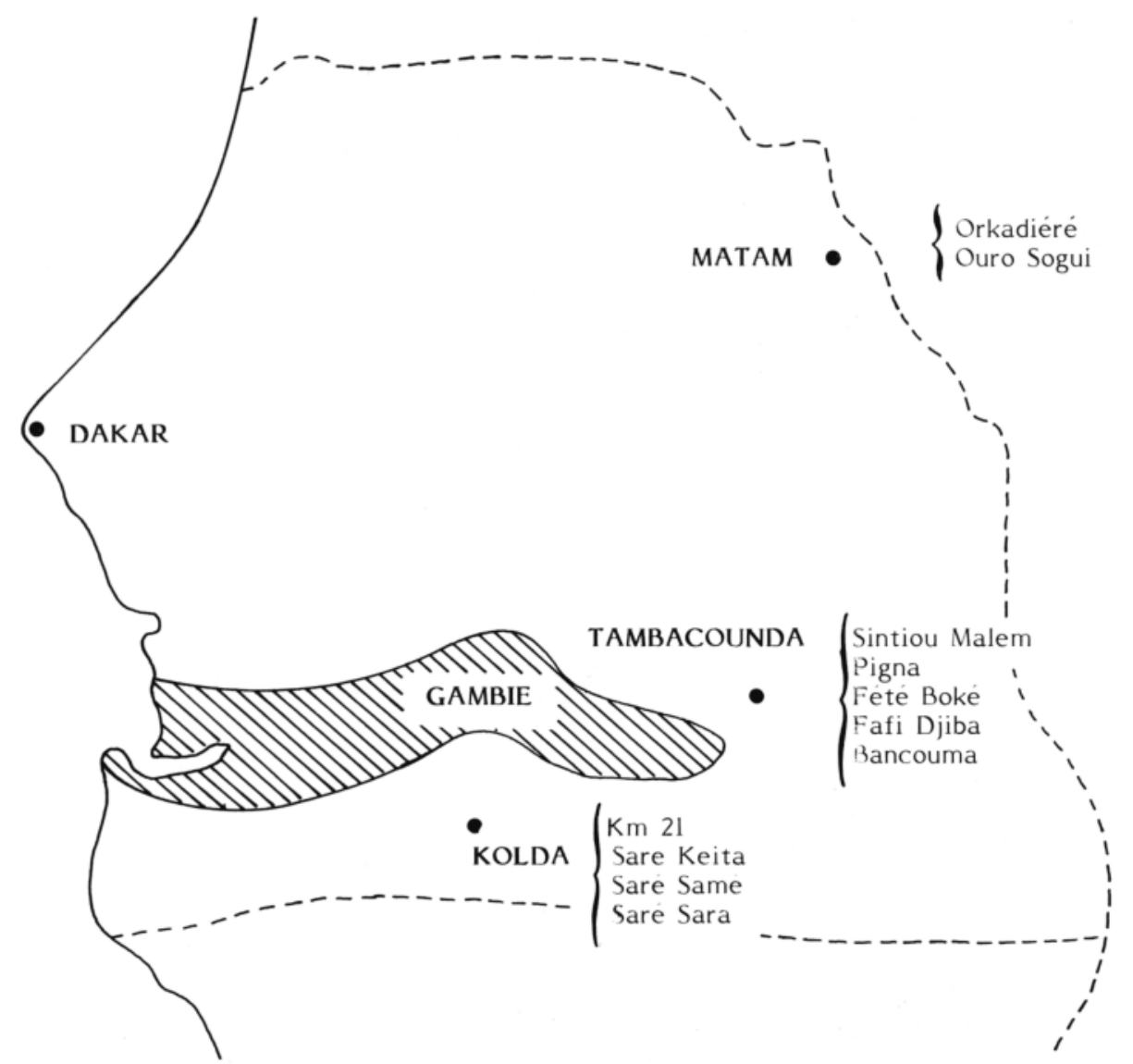

FIG. I. - Stations prospectées.

\section{II - Souches analysées}

(tableau I)

Nous analyserons successivement :

A - des souches de références de provenances diverses;

$\mathrm{B}$ - des souches obtenues à partir de Mollusques naturellement infestés ;

$\mathrm{C}$ - des souches obtenues à partir de miracidiums provenant d'enfants bilharziens ;

$\mathrm{D}$ - des souches obtenues à partir de miracidiums provenant de foie de Bovidae.

A - Souches de références de provenances diverses

A.1 : Schistosoma haematobium étudié par J. Richard en 1971 ; la souche a été isolée du Maroc par le Professeur Capron et a été maintenue au laboratoire chez Bulinus truncatus; nous disposons de 24 cercaires imprégnées (nº: $729 \mathrm{Z}$ ). 
Tableau I. - Souches analysées

\begin{tabular}{|c|c|c|c|c|c|c|c|c|}
\hline Souche & $\mathrm{E} / \mathrm{N}$ & $\mathrm{AD}$ & Mollusque & Vertèbre & $\mathrm{E} / \mathrm{N}$ & $\mathrm{L} / \mathrm{l}$ & Détermination & Origine \\
\hline $\begin{array}{l}\text { A1 } \\
\text { A2 } \\
\text { A3 } \\
\text { A4 } \\
\text { A5 } \\
\text { A6 } \\
\text { A7 } \\
\text { A8 } \\
\text { A9 } \\
\text { A10 } \\
\text { B1 } \\
\text { B2 } \\
\text { B3 }\end{array}$ & $\begin{array}{l}E \\
E \\
E \\
E \\
E \\
E \\
E \\
E \\
E \\
E \\
N \\
N \\
N \\
N \\
N\end{array}$ & $\begin{array}{l}0,70 \\
0,99 \\
0,65 \\
1,36 \\
1,24 \\
1,35 \\
0,57 \\
1,24 \\
1,33 \\
1,41 \\
0,69 \\
0,99 \\
0,68 \\
0,87 \\
1,09\end{array}$ & $\begin{array}{l}\text { truncatus } \\
\text { rohlfsi } \\
\text { obtusispira } \\
\text { truncatus } \\
\text { truncatus } \\
\text { truncatus } \\
\text { Physopsis sp. } \\
\text { Physopsis sp. } \\
\text { forskalii } \\
\text { forskalii } \\
\text { senegalensis } \\
\text { umbilicatus } \\
\text { umbilicatus }\end{array}$ & \begin{tabular}{|l} 
Homme \\
Homme \\
Saccostomus \\
Mouton \\
Souris \\
Mastomys \\
Praomys \\
Praomys \\
Homme \\
Homme \\
Souris \\
Souris \\
Souris 1 \\
Souris 2 \\
Hamster \\
Avvicanthis \\
Mouton Dakar \\
Souris \\
Hamster \\
Mouton Dakar \\
Souris \\
Souris \\
Souris \\
Homme \\
Souris \\
Hamster \\
Homme \\
Homme \\
Souris \\
Souris \\
Homme \\
Mouton
\end{tabular} & $\begin{array}{l} \\
N \\
E \\
E \\
E \\
E \\
E \\
E \\
N \\
N \\
E \\
E \\
E \\
E \\
E \\
E \\
E \\
E \\
E \\
E \\
E \\
E \\
E \\
N \\
E \\
E \\
N \\
N \\
E \\
E \\
N \\
N\end{array}$ & $\begin{array}{l}- \\
= \\
= \\
\bar{Z} \\
3,28 \\
\overline{-} \\
\overline{-} \\
2,18 \\
2,34 \\
2,47 \\
2,47 \\
2,34 \\
2,44 \\
2,30 \\
= \\
\bar{Z} \\
2,25 \\
2,49 \\
2,01 \\
\overline{-} \\
2,47\end{array}$ & $\begin{array}{c}\mathrm{B} \\
\mathrm{B} \\
? \\
? \text { bovis } \\
\mathrm{A}+\text { ?B ou ?C } \\
\mathrm{A}+\text { ?B ou ?C } \\
\mathrm{A} \\
\mathrm{A} \\
\mathrm{B}+\mathrm{C}\end{array}$ & $\begin{array}{l}\text { Maroc } \\
\text { R.C.A. } \\
\text { Madagascar } \\
\text { Sardaigne } \\
\text { Soudan } \\
\text { Soudan } \\
\text { Afrique Sud } \\
\text { Botswana } \\
\text { Gabon } \\
\text { Gabon } \\
\text { Orkadiéré } \\
\text { Fété-Boké } \\
\\
\text { Pigna }\end{array}$ \\
\hline
\end{tabular}

A.2 : Schistosoma (?) haematobium - les œufs des urines d'un malade de République Centrafricaine ont infesté un élevage de Bulinus rohlfsi; nous disposons de 19 cercaires étudiées par Bayssade-Dufour, 1982 (nº : $313 \mathrm{PE}$ ).

A.3 : S. haematobium de Madagascar passé chez Bulinus obtusispira et le Rongeur Saccostomus campestris (32 passages) très aimablement communiqué par le Dr. Striebel du Laboratoire Pharmacologique de Genève; nombreuses cercaires disponibles (no: 72 TC).

A.4 : S. bovis étudié par J. Richard (1971); la souche a été isolée d'un Mouton de Sardaigne par le Professeur Biocca et a été maintenue en laboratoire chez Bulinus truncatus et Mouton; 11 cercaires disponibles (nº: 62 TD).

A.5 : S. bovis isolé d'un petit bétail (Mouton ou Chèvre) du Soudan; passé par Bulinus truncatus; nombreuses cercaires colorées par le Professeur Combes, aimablement communiquées par celui-ci ( $\left.\mathrm{n}^{\circ}: 62 \mathrm{TD}\right)$. 
A.6:S. bovis isolé d'un pool d'œufs récoltés dans les foies de Bœufs, Moutons et Chèvres dans les abattoirs de Kosti (Soudan), aimablement communiqué par le Professeur Webbe ; passé par Bulinus truncatus et Mastomys; cinq cercaires disponibles ( $\left.\mathrm{n}^{0}: 65 \mathrm{TD}\right)$.

A.7 : S. mattheei d'Afrique du Sud passé sur Physopsis sp. et Praomys natalensis ; aimablement communiqué par le Dr. Pitchford ; nombreuses cercaires ( ${ }^{\circ}: 263 \mathrm{PE}$ ).

A.8 : S. leiperi du Botswana passé chez Physopsis sp. et Praomys natalensis; aimablement communiqué par le Dr. Pitchford. 15 cercaires sont dessinées ( ${ }^{\circ}: 259 \mathrm{PE}$ ).

A.9 : S. intercalatum; les œufs proviennent des selles d'une Femme gabonaise; ils infestent un élevage de Bulinus forskalii. Nous disposons de 17 cercaires étudiées par Bayssade-Dufour et coll. (1980) (no: $111 \mathrm{PE}$ ).

A.10:S. intercalatum; les oufs proviennent des selles d'une Femme gabonaise; ils infestent un élevage de Bulinus forskalii. Nous disposons de 17 cercaires étudiées par Bayssade-Dufour (1982) (nº:320 PE).

\section{$\mathrm{B}$ - Souches obtenues à partir de Mollusques naturellement infestés}

B. 1 : Nombreuses cercaires issues de Bulinus senegalensis (33 TD) récoltés à Orkadiéré le 8-12-83 dans une mare assez grande $(100 \times 50 \mathrm{~m})$, sans végétation, au milieu du village.

Infestation de 2 Souris : l'une morte, un mois après l'infestation, ne contient que des Vers immatures; l'autre, sacrifiée au troisième mois, ne contient que des Schistosomes mâles.

B.2 : Nombreuses cercaires issues de Bulinus umbilicatus (3 TD) récoltés à Fété Boké le 28-11-83 dans une mare peu profonde, très vaseuse à $10 \mathrm{~km}$ environ au-delà de Mâyel Dibi sur la piste de Matam; de nombreuses empreintes d'animaux sont visibles autour de la mare.

Les cercaires sont mises en présence de six Souris blanches. Les autopsies, étagées $d u{ }^{\mathrm{e}}$ au $6^{\mathrm{e}}$ mois après l'infestation montrent que 2 Souris sont négatives, les quatres autres ont des Schistosomes de petite taille dans le parenchyme hépatique ; aucun œuf n'est observé.

B.3 : Très nombreuses cercaires issues de Bulinus umbilicatus récoltés dans la mare de Pigna (6 TD) à $25 \mathrm{~km}$ de Tambacounda. Il s'agit d'une mare à fond plat et dur, profonde d'environ $40 \mathrm{~cm}$, dont $10 \mathrm{~cm}$ de vase grise et $30 \mathrm{~cm}$ d'eau jaune mais transparente; végétation abondante (nénuphars). Bulins très abondants.

L'indice $\mathrm{AD}$ est pris en début d'expérience sur les cercaires d'un Mollusque. Certains Mollusques continuent à émettre des cercaires après 2 mois d'observation. 7 d'entre eux sont isolés et les indices $\mathrm{AD}$ sont pris pour les cetcaires émises par chacun d'eux séparément.

De nombreux Mammifères ont été infestés avec succès : 25 Souris blanches, 5 Hamsters, 4 Arvicanthis, 2 Moutons (1 à Dakar, 1 à Paris). 
Tous les animaux (sauf le Mouton de Paris, qui n'avait que des mâles) ont permis de recueillir des adultes mâles, femelles, et des œufs. Les œufs provenant des Arvicanthis n'ont pas été dessinés.

B.4 : Très nombreuses cercaires issues de Bulinus umbilicatus récoltées dans une mare à gauche de la route allant d'Ouro-Sogui à Matam les 7 et 8-12-83. Il s'agit d'une mare résiduelle à bords inclinés en pente douce, à fond vaseux, sans végétation. La pose de pièges (branches d'arbres) permet la capture de nombreux Mollusques.

Les animaux infestés avec les cercaires sont tous positifs et ont permis d'obtenir des adultes et des œufs. Le Mouton (Dakar) ne peut pas être pris en considération (un œuf dessiné). L'indice $\mathrm{L} / 1$ du Hamster ne repose que sur les dessins de 3 œufs.

B.5 : Cercaires obtenues à partir d'un seul Bulinus jousseaumei sur 3 récoltés à Saré Keita dans un marigot à la sortie de Kolda, route de Ziguinchor. Mollusques récoltés à droite du pont et à proximité de celui-ci, direction Ziguinchor. Eau assez profonde, végétation très abondante, en particulier nénuphars. Lieu très fréquenté par les Humains et les Bovins.

Cinq Souris ont été infestées; toutes sont positives, mais n'hébergent que des Schistosomes mâles.

B.6 : Cercaires obtenues à partir d'un seul Bulinus jousseaumei positif sur de nombreux spécimens récoltés dans un cours d'eau a proximité du pont situé à $21 \mathrm{~km}$ de Kolda, près du village de Saré Sara, sur la route de Velingara. Mollusques récoltés à proximité du pont en amont et en aval. Cours d'eau élargi par endroits, eau assez profonde (environ 1 mètre), végétation très abondante, Nénuphars et Pistias; ailleurs cours d'eau resserré, ombragé, sans végétation; léger courant. Mollusques sur branches mortes ; fréquentation humaine et bovine.

Cinq Souris ont été infestées. Les trois Souris autopsiées cinq mois plus tard n'ont que des Schistosomes mâles.

Les cercaires de ce Bulin ont des chétotaxies très anormales. Presque aucune d'entre elles n'a les 6 soies AD au complet et l'indice AD ne peut être déterminée.

B.7 : Cercaires obtenues à partir d'un seul Bulinus forskalii à Saré Keita, même gîte que B.5, mais Mollusques recueillis à gauche du pont en tournant le dos à Kolda.

Quatre Souris ont été mises en présence de cercaires; l'une des quatre, morte un mois et demi après l'infestation, a permis l'obtention de Schistosomes immatures dans le foie. Les trois dernières, autopsiées environ 4 mois après leur infestation, n'ont fourni aucun Ver.

\section{$\mathrm{C}$ - Souches expérimentales obtenues à partir d'urines humaines}

C. 1 : Miracidiums provenant d'urines d'enfants de Sintiou Malem.

Trois espèces de Bulins ont été expérimentées : $B$. forskalii et $B$. guernei restent négatifs ; $B$. umbilicatus s'infeste.

L'indice des cercaires est établi.

Un Hamster et 4 Souris sont infestés : des adultes et des œufs sont obtenus dans le foie de tous les animaux. 
C. 2 : Miracidiums provenant d'urines d'enfants d'Orkadiéré.

Trois espèces de Bulins ont été expérimentées : $B$. forskalii, B. guernei et $B$. umbilicatus.

B. umbilicatus s'infeste; l'indice des cercaires est établi.

C.3 : Miracidiums provenant d'urines d'enfants de Saré Samé.

Trois espèces de Bulins sont expérimentées. Elles sont positives à des degrés divers.

a) B. umbilicatus avec un bon taux d'infestation (27\%). L'indice $\mathrm{AD}$ des cercaires est établi.

b) B. guernei. 2 Mollusques sur 27 s'infestent et émettent quelques rares cercaires. Ces cercaires infestent une Souris blanche. Celle-ci, sacrifiée 2 mois $1 / 2$ plus tard, a des Schistosomes mâles immatures. ${ }^{1}$

c) B. jousseaumei. Les Mollusques s'infestent (55\%) et émettent des cercaires. Ces cercaires infestent 6 Souris blanches. Sacrifiées 2 mois $1 / 2$ plus tard, trois n'ont que des mâles, mais trois autres ont des couples d'adultes et des œufs dans le foie ; le dessin de ces œufs n'a pas été fait.

C.4 : Miracidiums provenant d'urines d'enfants de Saré Sara.

Quatre espèces de Bulins sont expérimentées : B. guernei, B. umbilicatus, $B$. forskalii et B. jousseaumei.

Cette dernière espèce, seule, est positive (45\% des Mollusques). L'indice AD est calculé.

Six Souris et quatre Hamsters sont mis en présence de cercaires : une Souris est négative; trois n'ont que des Schistosomes femelles; deux ont des couples et des œufs ; ces derniers n'ont pas été étudiés. Trois Hamsters sont négatifs, un est positif avec mâles et femelles, mais les oufs sont peu nombreux et rarement embryonnés.

\section{$\mathrm{D}$ - Souches obtenues à partir de miracidiums provenant de foie de Bovidae}

D. 1 : Un Mouton des abattoirs de Dakar a une riche infestation du foie. Trois oufs seulement sont mesurés. Les miracidiums infestent $B$. umbilicatus et $B$, jousseaumei. Les indices $\mathrm{AD}$ des cercaires sont établis pour chacun des deux lots.

\section{III - Résultats}

\section{A - Indice $A D$ des cercaires (tableau $I I$ )}

L'indice $\mathrm{AD}$, c'est-à-dire le rapport $\frac{\mathrm{AID}}{\mathrm{AIID}-\mathrm{AIID}}$ - AIIID tel qu'il est défini par J. Richard (1971) et Ch. Bayssade-Dufour (1982) a été calculé pour les souches disponibles du Sénégal, ou repris des résultats publiés précédemment par Bayșsade-Dufour.

I. Cette donnée mérite d'être vérifiée car les deux espèces se ressemblent et un spécimen de $B$. umbilicatus a pu, par erreur, être mis avec les $B$. guernei. 
Les 28 résultats dont nous disposons sont donnés sur le tableau $I I$. Ils permettent les constatations suivantes :

a) Les Mollusques $B$. umbilicatus spontanément infestés, récoltés à Pigna (B3), ont émis de nombreuses cercaires. Nous disposons de 8 indices AD. L'un a été effectué en décembre 1983 sur les cercaires émises par différents mollusques au moment de la récolte. Les 7 autres concernent les cercaires émises en février 1984 par 7 Mollusques isolés.

On constate qu'ils se répartissent en 3 groupes :

$A: n^{0} 4: 0,66 \pm 0,11 ; n^{0} 2: 0,67 \pm 0,14 ; n^{0} 3: 0,69 \pm 0,12$.

B : $n^{0} 1: 0,84 \pm 0,11 ;$ Déc. $83: 0,87 \pm 0,09 ; n^{0} 7: 0,88 \pm 0,14 ; n^{0} 5: 0,89 \pm 0,16$.

$\mathrm{C}: \mathrm{n}^{0} 6: 1,09 \pm 0,11$.

La comparaison des moyennes entre le $n^{0} 1$ (plus petit des B) et le $n^{0} 3$ (plus grand des A) montre une différence significative avec le risque $10 \%$ mais non avec le risque $5 \%$.

La comparaison des moyennes entre le $n^{0} 5$ (plus grand des B) et le $n^{\circ} 6$ (groupe C) montre également une différence significative avec le risque $10 \%$ mais non avec le risque $5 \%$.

TABLEAU II. - Indice AD des Cercaires.

\begin{tabular}{|c|c|c|c|}
\hline Provenance & Mollusque & Indice & Détermination \\
\hline Afrique du Sud & truncatus & $0,57 \pm 0,04$ & mattheei \\
\hline $\begin{array}{l}\text { Madagascar } \\
\text { Pigna (II-84) } \\
\text { Sintiou Malem } \\
\text { Pigna (II-84) } \\
\text { Saré Sara } \\
\text { Orkadiéré } \\
\text { Pigna (II-84) } \\
\text { Maroc } \\
\text { Saré Samé } \\
\text { Orkadiéré }\end{array}$ & $\begin{array}{l}\text { obtusispira } \\
\text { umbilicatus (4) } \\
\text { umbilicatus } \\
\text { umbilicatus (2) } \\
\text { jousseaumei } \\
\text { senegalensis } \\
\text { umbilicatus (3) } \\
\text { truncatus } \\
\text { umbilicatus } \\
\text { umbilicatus }\end{array}$ & $\begin{array}{l}0,65 \pm 0,17 \\
0,66 \pm 0,11 \\
0,67 \pm 0,20 \\
0,67 \pm 0,14 \\
0,69 \pm 0,11 \\
0,69 \pm 0,10 \\
0,69 \pm 0,12 \\
0,70 \pm 0,10 \\
0,71 \pm 0,17 \\
0,74 \pm 0,14\end{array}$ & $\begin{array}{c}\text { haematobium } \\
\text { A } \\
\text { A } \\
\text { A } \\
\text { A } \\
\text { A } \\
\text { A } \\
\text { haematobium } \\
\text { A } \\
\text { A }\end{array}$ \\
\hline $\begin{array}{l}\text { Pigna (II-84) } \\
\text { Pigna (XII-83) } \\
\text { Pigna (II-84) } \\
\text { Ouro Sogui } \\
\text { Pigna (II-84) } \\
\text { Dakar } \\
\text { Saré Keita } \\
\text { R.C.A. } \\
\text { Fété-Boké }\end{array}$ & $\begin{array}{l}\text { umbilicatus (1) } \\
\text { umbilicatus } \\
\text { umbilicatus (7) } \\
\text { umbilicatus } \\
\text { umbilicatus (5) } \\
\text { jousseaumei } \\
\text { jousseaumei } \\
\text { rohlfsi } \\
\text { umbilicatus }\end{array}$ & $\begin{array}{l}0,84 \pm 0,11 \\
0,87 \pm 0,09 \\
0,88 \pm 0,14 \\
0,88 \pm 0,15 \\
0,89 \pm 0,16 \\
0,90 \pm 0,15 \\
0,96 \pm 0,06 \\
0,99 \pm 0,10 \\
0,99 \pm 0,18\end{array}$ & $\begin{array}{l}\mathrm{B} \\
\mathrm{B} \\
\mathrm{B} \\
\mathrm{B} \\
\mathrm{B} \\
\mathrm{B} \\
\mathrm{B} \\
? \\
\mathrm{~B}\end{array}$ \\
\hline $\begin{array}{l}\text { Dakar } \\
\text { Pigna (II-84) }\end{array}$ & $\begin{array}{l}\text { umbilicatus } \\
\text { umbilicatus }(6)\end{array}$ & $\begin{array}{l}1,10 \pm 0,26 \\
1,09 \pm 0,11\end{array}$ & $\begin{array}{l}\mathrm{C} \\
\mathrm{C}\end{array}$ \\
\hline $\begin{array}{l}\text { Soudan } \\
\text { Botswana } \\
\text { Gabon } \\
\text { Soudan } \\
\text { Sardaigne } \\
\text { Gabon } \\
\text { Saré Keita }\end{array}$ & $\begin{array}{l}\text { truncatus } \\
\text { Physopsis sp. } \\
\text { forskalii } \\
\text { truncatus } \\
\text { truncatus } \\
\text { forskalii } \\
\text { forskalii }\end{array}$ & $\begin{array}{l}1,24 \pm 0,16 \\
1,24 \pm 0,12 \\
1,33 \pm 0,14 \\
1,35 \pm 4,44 \\
1,36 \pm 0,14 \\
1,41 \pm 0,20 \\
1,42 \pm 0,23\end{array}$ & $\begin{array}{l}\text { bovis } \\
\text { leiperi } \\
\text { intercalatum } \\
\text { bovis } \\
\text { bovis } \\
\text { intercalatum } \\
\text { (?) bovis }\end{array}$ \\
\hline
\end{tabular}


b) Les Mollusques $B$. umbilicatus et $B$. jousseaumei infestés expérimentalement à partir d'urines humaines $(\mathrm{C} 1, \mathrm{C} 2, \mathrm{C} 3, \mathrm{C} 4)$ émettent des cercaires qui appartiennent toutes au groupe A.

c) L'AD de S. mattheei sensu stricto d'Afrique du Sud est significativement plus petit que tous ceux du groupe $\mathrm{A}$. La valeur de ce résultat reste à vérifier puisque nous n'avons disposé que d'une seule souche et que la distance entre le groupe 1 et le groupe 2 est faible. Il faut noter en tous cas une très nette différence entre le groupe mattheei et le groupe B.

d) L'ensemble bovis, intercalatum, leiperi est homogène et se sépare clairement des autres espèces.

e) A l'exception des cercaires émises à Saré Keita par B. forskalii (vraisemblablement référables à $S$. bovis) toutes les souches isolées du Sénégal, quelle que soit leur origine, sont référables à l'un des 3 groupes $\mathrm{A}, \mathrm{B}$ ou $\mathrm{C}$.

\section{B - Indice L/l des aufs (tableau III)}

Ce travail était destiné primitivement à séparer les cercaires de $S$. haematobium de celles de $S$. bovis. Les œufs de ces 2 espèces sont très différents. C'est pourquoi l'étude morphologique des œufs des différentes souches n'a été entreprise que tardivement, lorsque le problème est apparu complexe. Nous ne disposons donc que de 8 indices $\mathrm{L} / 1$ portant sur au moins 10 mesures. D'autres chiffres, établis sur moins de 5 œufs, sont cependant significatifs, car les distances entre les moyennes sont très grandes.

Les résultats sont représentés sur les tableaux $I$ et III et la figure 2.
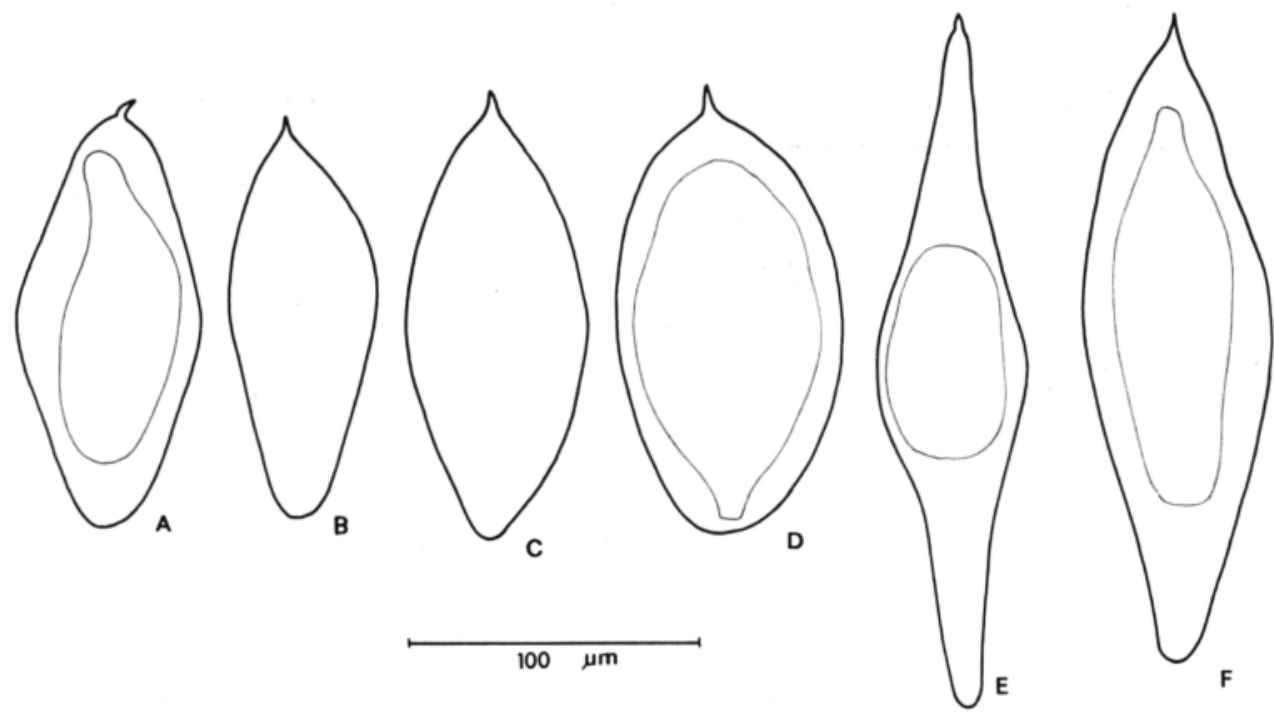

FIG. 2. - Eufs : A) Foie Mouton Dakar ; B) Foie Bcuf Tambacounda ; C) Urine Enfant Orkadiéré ; D) Urine Enfant Saré Samé ; E) Foie Bouf Dakar ; F) Souche mattheei adressée par le Dr. Pitchford. Les 6 œufs contiennent un miracidium (les contours n'ont pas été tracé en $B$ et C). 
Les remarques suivantes peuvent être faites :

a) Nous ne disposons que de 2 œufs ayant l'aspect typique de $S$. bovis (fig. $2 \mathrm{E}$ ). Ils proviennent du foie d'un Bœuf de l'abattoir de Dakar. Ils ont un indice de 4,4 mais cette donnée n'a évidemment aucune valeur statistique.

Cependant, les figures disponibles dans la littérature montrent que l'indice L/1 des œufs de bovis sensu stricto, supérieur à 4 , sépare sans aucune ambiguïté $S$. bovis des autres espèces en cause.

TABleau III. - Indice $L / 1$ des œufs.

\begin{tabular}{lccc}
\hline Provenance & Nb. mesures & Hôte & Indice \\
\hline Saré Samé & 12 & Homme & $2,01 \pm 0,14$ \\
Pigna & 31 & Souris 172 TD & $2,18 \pm 0,08$ \\
Sintiou Malem & $6+3+2$ & Souris & $2,19 \pm 0,12$ \\
Sintiou Malem & 10 & Homme & $2,25 \pm 0,11$ \\
Ouro Sogui & 22 & Souris & $2,33 \pm 0,06$ \\
Pigna & $8+12$ & Souris 156 TD & $2,35 \pm 0,12$ \\
Orkadiéré & 12 & Homme & $2,44 \pm 0,15$ \\
Pigna & 13 & Mouton-Dakar & $2,47 \pm 0,12$ \\
Ouro Sogui & 1 & Mouton Paris & 2,30 \\
Ouro Segui & 3 & Hamster & $2,44 \pm 0,11$ \\
Pigna & 4 & Hamster & $2,47 \pm 0,40$ \\
Abatt. Dakar & 3 & Mouton & $2,47 \pm 0,10$ \\
Abatt. Dakar & 4 & Mouton & $2,61 \pm 0,41$ \\
Abatt. Tambac. & 4 & Bœuf & $2,79 \pm 0,22 ?$ \\
Souche mattheei & 3 & Praomys & $3,28 \pm 0,62$ \\
Bovis Dakar & 2 & Bœuf & $4,4 \pm ?$ \\
\hline
\end{tabular}

b) Nous disposons seulement de 3 œufs de mattheei sensu stricto.

La valeur trouvée, $3,28+0,62$, bien que très peu précise, est bien différente de celles des œufs du Sénégal. Elle est du même ordre que celle déduite des mesures des œufs de Veglia et Le Roux (1929).

c) Eufs des urines humaines.

Trois mesures sont disponibles : - celle de Saré-Samé (Casamance) à 2,01 $\pm 0,14$; - celle de Sintiou Malem (Est) à 2,25 \pm 0,11; - celle de Orkadiéré (Nord-Est) à $2,44 \pm 0,15$.

Il y a une différence significative dans la comparaison des moyennes avec un risque de $5 \%$ entre Saré-Samé et Sintiou Malem, avec un risque de $10 \%$ entre Sintiou Malem et Orkadiéré.

Donc avec l'indice L/l, comme avec l'indice AD, les Schistosomes du Sénégal se révèlent hétérogènes.

d) Il y a une différence significative avec un risque à $5 \%$ entre l'Homme de Saré-Samé et l'indice le plus petit du groupe suivant (Souris 172 TD de Pigna). 
Les autres indices (entre 2,18 et 2,47) n'offrent pas de hiatus nettement prononcé. Il est possible pourtant que le groupe $2,35-2,47$ soit homogène et que le groupe 2,18-2,25 fasse transition avec Saré-Samé.

e) Dans les 3 couples Souris-Homme (Sintiou Malem), Souris-Mouton (Pigna), Souris-Hamster (Ouro-Sogui), on constate que le rapport L/I de la Souris est un peu plus faible que celui de l'autre Mammifère (Homme, Mouton ou Hamster) infesté par la même souche.

f) Deux souris infestées avec les cercaires de Pigna émettent des œufs qui ont des indices dont les moyennes sont significativement différentes avec un risque de $10 \%$. L'hétérogénéité des indices AD des cercaires du gite se retrouve donc au niveau des Schistosomes adultes de la Souris.

\section{$\mathrm{C}$ - Conséquences taxonomiques découlant de ces deux indices}

Les Bulins du Sénégal hébergent dans certains gîtes 3 types de cercaires ayant des indices $\mathrm{AD}$ différents.

Les 3 indices indiquent en outre qu'il ne s'agit ni de $S$. mattheei ni de $S$. bovis.

Le type A (indice de 0,65 à 0,74 ) doit correspondre à $S$. haematobium puisqu'il coïncide avec l'indice $\mathrm{AD}$ des souches de référence déjà étudiées.

Les types $\mathrm{B}$ et $\mathrm{C}$ appartiennent à une ou à deux autres espèces. La discussion qui va suivre amène à la conclusion que le taxon $S$. curassoni doit être rétabli pour désigner cette espèce ou l'une de ces deux espèces.

J'indice $\mathrm{L} / \mathrm{l}$ de son côté ne correspond au typique $S$. haematobium que lorsqu'il est aux environs de 2,0. Schistosoma haematobium est donc présent au Sénégal, en particulier chez l'Homme dans le Sud, mais, dans la plupart des prélèvements, l'indice est aux environs de $2,30-2,50$; cela correspond semble-t-il à une autre espèce qu'haematobium, qui n'est ni bovis, ni mattheei et qui est donc très vraisemblablement curassoni.

Pour simplifier l'exposé, nous pensons donc, dans la suite du texte, pouvoir utiliser les termes d'haematobium et de curassoni.

\section{$\mathrm{D}$ - Données diverses}

\section{1 - Indice $U$}

Richard (1971), puis Bayssade-Dufour (1982) ont tenté de séparer les cercaires de Schistosomes par la composition du groupe de papilles situées ventralement UV et dorsalement UD au quart postérieur du tronc caudal.

- le groupe haematobium et margreboreiei semble porter 4 papilles ventrales et 4 papilles dorsales.

- le groupe bovis, mattheei, leiperi et intercalatum semble porter 6 papilles ventrales et 6 papilles dorsales.

Cet indice $\mathrm{U}$ est très difficile à utiliser, car la limite du groupe de papilles prises en considération prête à discussion. 
A titre provisoire, et d'après les documents actuellement analysés, il semble bien que les cercaires déterminées curassoni soient de type 6 comme mattheei et bovis, et non de type 4 comme haematobium.

\section{2 - Mollusques sensibles}

Si les déterminations fondées sur les indices $\mathrm{AD}$ des cercaires ou $\mathrm{L} / 1$ des œufs sont exactes et si les infestations expérimentales ne sont pas faussées par une infestation préexistante des Mollusques:

- S. curassoni a été trouvé en infestation naturelle chez B. umbilicatus à Pigna, à Ouro Sogui, à Fété Boké, et chez B. jousseaumei à Saré Keita. L'infestation expérimentale a été obtenue chez $B$. umbilicatus.

- $S$. haematobium a été trouvé en infestation naturelle chez $B$. senegalensis à Orkadiéré et chez $B$. umbilicatus à Pigna. L'infestation expérimentale a été obtenue chez B. umbilicatus, (?) B. guernei et B. jousseaumei.

- $S$. bovis a été trouvé en infestation naturelle chez $B$. forskalii à Saré Keita.

\section{3 - Vertébrés sensibles}

Si, comme dans le paragraphe précédent, l'on admet que les déterminations fournies par les indices $\mathrm{AD}$ ou $\mathrm{L} / 1$ sont valables et que l'expérimentation a échappé aux infestations spontanées :

- S. curussoni a été trouvé en infestation naturelle chez l'Homme à Sintiou Malem et à Orkadiéré, chez le Mouton aux abattoirs de Dakar, chez le Bœuf aux abattoirs de Tambacounda. L'infestation expérimentale est obtenue chez les Souris, les Hamsters, les Arvicanthis et le Mouton. On obtient des couples fertiles chez tous ces animaux.

- S. haematobium a été trouvé en infestation naturelle pure chez l'Homme à Saré Sara, Saré Samé et associé à curassoni à Sintiou Malem et à Orkadiéré. Quelques infestations expérimentales positives semblent avoir été obtenues chez des Souris blanches et des Hamsters, mais les œufs n'ayant pas été mesurés, les expériences ne sont pas utilisables.

- S. bovis a été trouvé en infestation naturelle chez un Bœuf de Dakar. Les infestations expérimentales des Souris sont négatives ou ne donnent que des Vers immatures.

\section{IV - Données nouvelles}

Dans l'état actuel de nos recherches, les résultats exposés précédemment aboutissent aux conclusions suivantes:

1 - L'analyse morphologique des œufs montre que les formes mûres rencontrées dans les infestations naturelles ou expérimentales ne se répartissent pas en deux groupes : $S$. haematobium et $S$. bovis, mais en trois groupes : ce troisième groupe a un rapport L/1 d'environ 2,2 - 2,5 et non de 2,0 comme $S$. haematobium, ou de 4,4 comme S. bovis. 
Le Schistosome correspondant à cet œuf est, dans notre enquête, l'espèce dominante, dans le Nord-Est et dans l'Est, chez l'Homme et chez les Bovidés domestiques (Bœuf, Mouton).

S. haematobium sensu stricto se trouve surtout dans le Sud du pays (Casamance). Il n'existe que chez l'Homme.

S. bovis paraît de plus en plus rare. Il n'est trouvé que chez le Bœuf et chez (?) B. forskalii.

Les travaux précédemment menés dans ces régions nous font conclure que cette $3^{\mathrm{e}}$ espèce correspond à $S$. curassoni (voir chapitre V).

2 - Le Mollusque le plus fréquemment contaminé dans les conditions naturelles est $B$. umbilicatus, qui paraît être le principal vecteur de $S$. curassoni et de $S$. haematobium.

Précisons à ce propos que $B$. umbilicatus, qui n'a été séparé des espèces proches qu'à une date récente, n'avait jamais été signalé comme vecteur de Bilharziose. Il se révèle un excellent vecteur : il peut émettre plus de 1000 cercaires par Mollusque au cours d'une même émission provoquée expérimentalement, et son infestation naturelle est exceptionnellement longue (du 28-11-83 au 12-3-84 dans l'expérience B3).

3 - L'indice $\mathrm{AD}$ des cercaires de $S$. bovis n'est pas différent de celui de $S$. intercalatum et de celui de $S$. leiperi; il est situé entre 1,24 et 1,41 .

L'indice $\mathrm{AD}$ des cercaires de $S$. curassoni $(0,84$ à 0,99$)$ semble un peu plus grand que celui de $S$. haematobium $(0,65-0,74)$, qui semble lui-même un peu plus grand que celui de $S$. mattheei $(0,57)$.

\section{V - Discussion}

Certaines des données nouvelles indiquées ci-dessus sont inattendues, mais l'étude bibliographique des travaux effectués dans ces régions montre que ces données sont parfaitement compatibles avec celles de nos prédécesseurs.

Au sujet de cette Bilharziose du bétail provoquée par un Schistosome qui n'est pas $S$. bovis, nous disposons en Afrique occidentale de plusieurs données importantes :

Brumpt, 1931, décrit chez un Bœuf de Bamakou, Schistosoma curassoni sp. inquir., d'après les adultes et les œufs intra-utérins. Il indique " œufs un peu plus allongés que ceux du $S$. haematobium et du $S$. indicum, mais très voisins de ceux du $S$. mattheei mesurant de 110 à $120 \mu$ sur 30 à $40 \mu$ "; il indique que la détermination définitive ne pourra être faite que le jour où l'œuf mûr sera connu.

Grétillat (1962a et c) admet la validité de Schistosoma curassoni et montre sa grande fréquence au Sénégal et en Mauritanie chez les Bœufs, Moutons et Chèvres. Pour lui, les cufs de $S$. haematobium mesurent 115 à $185 \mu$ sur 64 à $78 \mu$ et ceux de curassoni 110 à $120 \mu$ sur 30 à $38 \mu$. Il infeste expérimentalement " $B$. guernei " et " $B$. truncatus rohlfsi".

Le même auteur (1962b), à partir des cufs d'urines d'enfants de la région de Dakar, infeste expérimentalement les deux mêmes espèces de Mollusques et inocule 
300 furcocercaires à un jeune Ovin, qui meurt au bout de cinq semaines avec 150 Schistosomes immatures. L'auteur conclut à l'existence d'une zoonose commune à l'Homme et aux Ruminants domestiques, qu'il nomme "Bilharziose ouest-africaine ».

Cette notion nouvelle n'est généralement pas admise. En effet, S. Grétillat n'a pas pu voir les œufs obtenus chez le Mouton contaminé avec des cercaires d'origine humaine, puisque les Vers obtenus sont immatures. Sa conviction qu'il s'agit de curassoni et non d'haematobium est fondée sur une notion erronée : il pense pouvoir distinguer $S$. curassoni des autres Schistosomes parce que le stade sporocyste serait absent chez curassoni (genre Proschistosoma) et présent chez les autres Schistosomes.

Capron et collaborateurs (1965) ont relevé ces erreurs, mais retiennent néanmoins que les travaux de Grétillat " relatant la transmission réussie d'une souche de $S$. haematobium d'origine humaine à de jeunes ovins, peuvent éclairer d'un jour nouveau l'épidémiologie de la Bilharziose à $S$. haematobium dans l'Ouest-Africain ".

Par ailleurs, les travaux de Capron et collaborateurs sont particulièrement intéressants à notre point de vue, car ils sont amenés à distinguer clairement deux types de souches d'haematobium: les souches algériennes et marocaines d'une part, et les souches mauritaniennes et sénégalaises d'autre part.

En effet, les souches algériennes et marocaines sont bien transmises par B. truncatus (originaire de Corse) et par "B. truncatus rohlfsi") (originaire du Tchad) ; par contre, les souches mauritaniennes et sénégalaises évoluent médiocrement chez "B.t. rohlfsi " du Tchad et pas du tout chez B. truncatus de Corse.

Il en est de même au Ghana où l'une des 2 souches de $S$. haematobium est transmise par B. truncatus rohlfsi et l'autre par B. globosus (Chu et coll., 1978). Il faut rappeler aussi qu'au Cameroun où il existe S. haematobium transmis par B. truncatus rohlfsi, et S. intercalatum transmis par B. forskalii, Southgate et coll. (1976) ont trouvé un hybride dont les œufs ont des caractères intermédiaires entre ceux des espèces parentales et infestent les deux espèces de Mollusques.

Grétillat (1961), a, comme nous-mêmes, travaillé dans la région de Tambacounda. Il est donc hors de doute que la plupart des Mollusques qu'il désigne comme $B$. guernei correspond à ce que nous nommons actuellement $B$. umbilicatus.

Il apparaît donc en conclusion, qu'à condition de faire les corrections taxonomiques nécessaires, nos résultats confirment ceux de nos prédécesseurs :

Le Mouton héberge dans le Nord et dans l'Est un Schistosome qui n'est ni bovis, ni mattheei, et que nous nommons curassoni. Par ailleurs, on trouve fréquemment, dans les urines des enfants, un œuf différent de celui d'haematobium, et qui a la même morphologie que celui de curassoni.

\section{VI - Conclusions}

Plusieurs difficultés subsistent dans l'interprétation des résultats.

a) en $\mathrm{C} 1$ et $\mathrm{C} 2$, les œufs dessinés dans les urines sont de type curassoni. Les Mollusques infestés avec les urines d'enfants de la même école ont émis (au moins partiellement) des cercaires de type haematobium. 
b) en $\mathrm{Cl}$, inversement, ce lot de Mollusques qui avait été considéré comme infesté par haematobium, a transmis aux souris une infestation de type B. curassoni.

Ces constatations ne sont pas très surprenantes puisque l'on sait que les 3 types de cercaires $A, B$ et $C$ existent dans un même gîte, que tel ou tel hôte peut servir de filtre en faveur de telle ou telle espèce, qu'une partie seulement des matériaux disponibles (œufs des urines ou cercaires des Mollusques) est examinée et que de toutes façons, nos Mollusques peuvent avoir une infestation spontanée non détectée.

Le travail comporte donc actuellement une partie aléatoire. Cependant plusieurs faits sont nettement établis :

a) Au Sénégal, les œufs de Schistosomes trouvés dans les urines des enfants sont différents d'une région à l'autre.

Ils sont de type haematobium $(\mathrm{L} / \mathrm{l}=2,0)$ dans le Sud, en Casamance.

Ils sont de type curassoni $(\mathrm{L} / \mathrm{I}=2,44)$ dans le Nord, à Orkadiéré.

Un type intermédiaire est trouvé dans l'Est à Sintiou Malem $(\mathrm{L} / \mathrm{l}=2,25)$.

b) Les Moutons n'ont que des œufs de type curassoni, différents de ceux de mattheei et de ceux de bovis.

c) Les indices $\mathrm{AD}$ des cercaires sont de 3 types :

- type A $(0,65$ à 0,74$)$ référable à haematobium.

- type $\mathrm{B}(0,84$ à 0,99$)$ référables à (?) curassoni.

- type $C$ (env. 1,10) référable à (?) curassoni.

En ce qui concerne ces types $B$ ou $C$, trois hypothèses peuvent être proposées :

a) le type $\mathrm{C}$ correspond à curassoni et le type $\mathrm{B}$ à une espèce nouvelle.

b) le type $\mathrm{C}$ correspond à curassoni et le type $\mathrm{B}$ à un éventuel hybride curassonihaematobium.

c) les types $\mathrm{B}$ et $\mathrm{C}$ ne méritent pas d'être séparés et l'ensemble correspond à une seule espèce : $S$. curassoni.

De toute façon l'indice $\mathrm{AD}$ de ces cercaires est différent de celui de mattheei $(0,57)$ et de celui du groupe bovis $(1,24$ à 1,42$)$.

Cet ensemble de faits est donc en accord avec plusieurs points importants publiés précédemment par Grétillat.

Remerciements. Ce travail a reçu le support financier de l'UNDP/World Bank/ WHO Special Programme for Research and Training in Tropical Diseases.

Nous remercions également MM. Combes, Pitchford, Striebel et Webbe qui nous ont fourni quelques-unes des souches de références utilisées ainsi que MM. Frandsen et Brown qui ont bien voulu effectuer la détermination des Mollusques.

Nous remercions bien vivement MM. Capron, Mac Cullough et Mott d'avoir bien voulu relire le manuscrit. 


\section{BIBLIOGRAPHIE}

Bayssade-Dufour Ch. : Chétotaxies cercariennes comparées de dix espèces de Schistosomes, Ann. Parasitol. Hum. Comp., I982;, 57, 467.

Brumpt E. : Description de deux Bilharzies de Mammifères africains : Schistosoma curassoni sp. inq. et Schistosoma rodhaini n, sp. Ann. Parasitol. Hum. Comp., r931, 9, 325.

Capron A., Deblock S., Biguet J., Clay A., Adenis L., Vernes A. : Contribution à l'étude expérimentale de la bilharziose à Schistosoma haematobium. Bull. Org. Mond. Santé, 1965,

CHU ${ }^{32,75 .}$ K. Y., KPO H. K., KLUMPP R. K. : Mixing of Schistosoma haematobium strains in Ghana. Bull. Wld Hlth Org., 1978, 56, 601.

Combes C., Bayssade-Dufour C., Cassone J. : Sur l'imprégnation et le montage des cercaires pour l'étude chétotaxique. Ann. Parasitol. Hum. Comp., I976, 5I, 399.

Grétillat S. : Epidémiologie de la bilharziose vésicale au Sénégal oriental. Bull. Org. Mond. Santé, I96I, 25,459 .

GRÉTILLAT S. : Recherches sur le cycle évolutif du Schistosome des Ruminants domestiques de l'Ouest-Africain (Schistosoma curassoni Brumpt, I93I). C.R. Acad. Sci. Paris, I962a, 25 5, I657.

Grérillat S. : Une nouvelle zoonose, la "Bilharziose Ouest-Africaine " à Schistosoma curassoni Brumpt, I93I, commune à l'Homme et aux Ruminants domestiques. C.R. Acad. Sci., Paris, I $962 \mathrm{~b}, 255, \mathrm{r} 805$.

Grérillat S. : Étude du cycle évolutif du Schistosome des Ruminants domestiques de 1'Ouest Africain et confirmation de l'espèce Schistosoma curassoni Brumpt, I93I. Ann. Parasitol. Hum. Comp., I $962,37,556$.

PITCHFORD R. J.: Differences in the egg morphology and certain biological characteristics of some African and Middle Eastern Schistosomes, genus Schistosoma, with terminal-spined eggs. Bull. Wld. Hith Org., 1965, 32, 105.

RICHARD J.: La chétotaxie des cercaires, valeur systématique et phylétique. Mem. Mus. Natn. Hist. Nat., sér. A, Zool., 67, I 79 p.

Schwartz D. : Methodes statistiques à l'usage des médecins et des biologistes. Flammarion, Paris, $1963,318 \mathrm{p}$.

Southgate V. R., WIJK H. B. VAN, WRIght C. A. : Schistosomiasis at Loum, Cameroun ; Schistosoma haematobium, $S$. intercalatum and their natural hybrid. $Z$. Parasitenk., I976, 49, I 45.

Veglia F., Le Roux P. L. : On the morphology of a Schistosome (Schistosoma mattheei, sp. nov.) from the sheep in the Cape Province. I sth Annual Rep. Divector Veterinary Services Union of South Africa, Prétoria, 1929, I, 335.

\section{Addenda}

Entre la note préliminaire à ce travail (Ann. Parasitol. Hum. Comp. 1984, 59, $527-$ 528), parue le 23 octobre 1984, et la correction de ces épreuves, plusieurs publications admettent également la validité de S. curassoni : Southgate V.R., Rollinson D., Vercruysse J., Trans. Roy. Soc. Trop. Med. Hyg., 1984, 78, correspondence, 848-849. - Vercruysse J., Southgate V.R., Rollinson D., Jour. Nat. Hist., 1984, 18, 969-976. 